\title{
Fluidez Verbal Semántica y Fonológica en una Muestra de Niños de Argentina
}

\author{
Julieta Fumagalli ${ }^{1}$ \\ CONICET - Universidad de Buenos Aires, Facultad de Filosofía y Letras, \\ Instituto de Lingüistica, Buenos Aires, Argentina \\ Federico Soriano \\ Instituto de Neurociencias Cognitiva y Traslacional (INCyT), Buenos Aires, Argentina \\ Diego Shalóm \\ CONICET, IFIBA, Facultad de Ciencias Exactas y Naturales, Universidad de Buenos Aires \\ Juan Pablo Barreyro \\ CONICET - Departamento de Investigación en Procesos Básicos, \\ Instituto de Investigaciones Facultad de Psicología/UBA, Buenos Aires, Argentina \\ María Macarena Martínez-Cuitiño \\ Laboratorio de Investigaciones en Lenguaje, Instituto de Neurociencia Cognitiva \\ y Traslacional (INCyT), Ineco/Favaloro/Conicet, Buenos Aires, Argentina
}

\section{Resumen}

El objetivo del presente trabajo es obtener información del patrón evolutivo de desempeño en tareas de fluidez verbal para una muestra de niños argentinos de nivel primario de $3^{\circ}, 5^{\circ}$ y $7^{\circ}$ grado. Se evaluó a los participantes con una tarea de fluidez semántica, en la que se evaluaron las categorías de animales, frutas y verduras, y partes del cuerpo para el dominio de seres vivos, y medios de transporte, instrumentos musicales y ropa para el de objetos inanimados. Asimismo fueron evaluados con una tarea de fluidez fonológica en la que se testearon los fonemas $/ \mathrm{f} /, / \mathrm{a} / \mathrm{y} / \mathrm{s} /$. Los datos obtenidos dan cuenta de diferencias de rendimiento entre las tareas de fluidez con un mejor desempeño en las tareas de fluidez semántica. Asimismo, en las tareas de fluidez semántica se observa un mejor rendimiento en el dominio de seres vivos. Las diferencias obtenidas en ambas tareas se identifican al comparar los grupos según escolaridad, siendo únicamente los niños de $3^{\circ}$ grado los que, con menor cantidad de ejemplares recuperados, se disocian significativamente de $\operatorname{los}$ de $5^{\circ}$ y $7^{\circ}$ grado. Estos hallazgos son discutidos a la luz de posibles diferencias en función del desarrollo cognitivo, de las funciones ejecutivas y del sistema semántico.

Palabras claves: Fluidez semántica, fluidez fonológica, escolaridad, niños.

\section{Fluência Semântica e Fonológica em uma Amostra de Crianças Argentinas}

\section{Resumo}

No presente trabalho oferecemos dados de tarefas de fluência verbal para crianças argentinas de $3^{\circ}, 5^{\circ} \mathrm{e}$ $7^{\circ}$ ano do ensino fundamental. Os participantes foram avaliados com uma tarefa de fluência semântica, que testou as categorias de animais, frutas e legumes e partes do corpo para o domínio dos seres vivos e meios de transporte, instrumentos musicais e roupas para os objetos inanimados. As crianças também foram avaliadas com uma tarefa de fluência fonológica que testou os fonemas /f /, /a/ e / s /. Os dados

Dirección para correspondencia: 25 de Mayo, 217/221, $1^{\circ}$ piso, (C1002ABE) Ciudad Autónoma de Buenos Aires, Buenos Aires, Argentina. Teléfono: (+54 11)4342-9710/9718, int. 103. E-mail: fumagallijulieta@gmail.com 
obtidos apontam para diferenças de desempenho entre as tarefas e mostraram um melhor desempenho nas de fluência semântica. Além disso, nas tarefas de fluência semântica os participantes tiveram um melhor desempenho no domínio dos seres vivos. Tanto para fluência semântica quanto fonológica, as diferenças estiveram presentes quando os grupos foram comparados pelo nível de escolaridade, de modo que os alunos de $3^{\circ}$ ano se dissociam significativamente dos $5^{\circ}$ e $7^{\circ}$ anos, produzindo menor quantidade de exemplares. Os resultados são discutidos à luz de possíveis diferenças originadas no desenvolvimento cognitivo, as funções executivas e o sistema semântico.

Palavras-chave: Fluência semântica, fluência fonológica, nível de escolaridade, crianças.

\title{
Phonological and Semantic Verbal Fluency Tasks in a Sample of Argentinean Children
}

\begin{abstract}
The aim of this work is to obtain data about the evolutionary pattern of performance in verbal fluency tasks for a sample of Argentinean primary school aged children $\left(3^{\text {rd }}, 5^{\text {th }}\right.$ y $7^{\text {th }}$ grade $)$ in semantic and phonological verbal fluency tasks. For the semantic fluency task, children were assessed in different categories: animals, fruits and vegetables, and body parts for living things domain, and means of transport, musical instruments and clothes for inanimate objects. For the phonological fluency assessment, children were tested with /f/, /a/ and /s/ phonemes. Both fluency tasks showed differences between grades, indicating the influence of education. Besides, irrespective of the grade, children performed better on the semantic fluency tasks. Results in the semantic fluency task also showed a better performance for the living things domain over the inanimate objects domain. Further analysis on the results showed that $3^{\text {rd }}$ grade children have the worst performance, compared to $5^{\text {th }}$ and $7^{\text {th }}$ grade children. The results of our work are discussed taking in account cognitive mechanisms, executive functions and semantic memory development.
\end{abstract}

Keywords: Semantic fluency, phonological fluency, educational status, children.

Las medidas de fluencia verbal son frecuentemente utilizadas tanto en investigación neuropsicológica como en la práctica clínica debido a la facilidad y rapidez para su toma, así como también dado el alto grado de fiabilidad que representan sus resultados frente a diversas patologías (Fernández, Marino, \& Alderete, 2004; Henry \& Crawford, 2004; Henry, Crawford, \& Phillips, 2004). En una prueba de fluidez verbal se les pide a los examinados que recuperen la mayor cantidad de ejemplares posibles para una o varias categorías en un tiempo determinado, por ejemplo en un minuto (Lezak, Howieson, \& Loring, 2004). Existen dos variantes de la prueba de fluencia verbal: la fluencia verbal semántica (FVS) y la fluencia verbal fonológica (FVF). En la primera variante, el evaluado debe recuperar la mayor cantidad ejemplares de una categoría semántica específica (por ejemplo, animales o frutas). En una prueba de FVF, se les pide a los evaluados que indiquen la mayor cantidad de palabras posibles que comienzan con un sonido determinado (por ejemplo, $/ \mathrm{p} / \mathrm{o} / \mathrm{s} /$ ).

En las tareas de fluencia verbal se ponen en juego diversos mecanismos cognitivos para llevar adelante la tarea: memoria de trabajo, atención sostenida, funciones ejecutivas, memoria semántica, estrategias de búsqueda y recuperación de ítems léxicos, entre otros. Por esto, se considera que las fluencias verbales son medidas muy sensibles a la hora de evaluar la plasticidad cognitiva y las estrategias de organización (Henry \& Crawford, 2004; Lezak et al., 2004; Stuss et al., 1998). Si bien ambas tareas exigen demandas ejecutivas (Hirshorn \& Thompson-Schill, 2006), en la FVS la recuperación de palabras se fundamenta a partir de las asociaciones semánticas y del significado de cada una de ellas, en tanto 
que la FVF implica estrategias que no se realizan habitualmente y requieren de mayor esfuerzo (Hurks et al., 2006).

Tanto la FVS como la FVF son herramientas útiles para el diagnóstico de ciertas patologías neurológicas. Los resultados en FVS son altamente sensibles para la pesquisa de enfermedades degenerativas (Lezak et al., 2004). Los puntajes en este tipo de tarea se utilizan para diagnosticar etapas tempranas de la enfermedad de Alzheimer y son un predictor importante de la enfermedad de Parkinson y de otros tipos de demencias como la demencia vascular, la variante semántica de la Demencia Frontotemporal o la Demencia subcortical, asimismo son útiles para identificar pacientes con Enfermedad de Huntington o aquellos con padecimientos psiquiátricos como la esquizofrenia (Binetti et al., 1995; Chertkow \& Bub, 1990; Galaverna, Marino, \& Abraham, 2008; Hodges \& Patterson, 1997; Rosser \& Hodges, 1994). Específicamente los pacientes fallarán cuando tengan problemas para sostener la atención por dificultades en el lóbulo frontal o cuando no puedan recuperar los ejemplares por alteraciones en el lóbulo temporal. Los pacientes con lesiones focales en el lóbulo frontal presentan dificultades en la tarea de FVF, ya que no pueden utilizar de manera adecuada los mecanismos cognitivos necesarios para realizar las búsquedas, iniciar las respuestas y realizar el monitoreo. Los pacientes con dificultades en el lóbulo temporal tendrán problemas con la FVS puesto que no podrán seleccionar ni agrupar adecuadamente los ejemplares de las diferentes categorías evaluadas por dificultades en la memoria semántica (Troyer, Moscovitch, Winocur, Alexander, \& Stuss, 1998).

Diversos estudios han demostrado que existen variables sociodemográficas que inciden en el desempeño de la prueba, siendo la edad y el nivel educativo alcanzado por los sujetos las dos variables más importantes (Kempler, Teng, Dick, Taussig, \& Davis, 1998). Algunas investigaciones han demostrado que los resultados en las pruebas de fluencia verbal mejoran proporcionalmente con el avance de la edad (Casals-Coll et al., 2013; Fernández et al., 2004; Kempler et al., 1998; Lezak et al., 2004; Lozano Gutiérrez \& Ostrosky-Solís, 2006; Ollari \&
Diez, 2005; Pekkala et al., 2009). Sin embargo, existen estudios con adultos mayores que muestran la existencia de un punto de inflexión (pasada la sexta década de vida) a partir del cual el desempeño comienza a decrecer a medida que la edad aumenta. Incluso, se ha demostrado que el desempeño decaería más en hombres que en mujeres (Rodríguez-Aranda \& Martinussen, 2006). La escolarización de los sujetos es especialmente importante para las pruebas de FVF (Ratcliff et al., 1998). Otra variable sociodemográfica a tener en cuenta es el sexo. Los estudios disponibles encuentran resultados contradictorios al respecto. En algunos casos, el género representó una variable significativa en la distribución de los resultados (Capitani, Laiacona, \& Barbarotto, 1999; Laws, 1999, 2004; Marino \& Alderete, 2010; Marra, Ferraccioli, \& Gainotti, 2007; McKenna \& Parry, 1994; Soriano et al., 2015), mientras que en otros no tuvo ninguna incidencia o esta fue relativa (Casals-Coll et al., 2013; Fernández et al., 2004). En general en tareas de fluencia verbal se identifica un mejor desemepeño de las mujeres en relación con los hombres (Halpern, 1992). En lo que respecta a la fluencia semántica los resultados encuentran diferencias pero, a grandes rasgos, las mujeres son mejores con las categorías de frutas/verduras y muebles, y los hombres con las categorías animales y herramientas (Albanese, Capitani, Barbarotto, \& Laiacona, 2000).

Las investigaciones con tareas de fluencia verbal hechas en hablantes nativos del español son escasas si las comparamos, por ejemplo, con las realizadas en una lengua como el inglés (Tombaugh, Kozak, \& Rees, 1999). Existen estudios realizados sobre población mexicana (Ostrosky-Solís \& Ardila, 1991), sobre población colombiana (Ramírez, Ostrosky-Solís, Fernández, \& Ardila-Ardila, 2005) y española (Buriel, Gramunt, Bohm, Rodes, \& Pena-Casanova, 2004; Villodre et al., 2006). Para el territorio argentino, existen algunos estudios específicos sobre el tema (Comesaña \& García Coni, 2013; Fernández et al., 2004; Marino \& Alderete, 2010; Villodre et al., 2006; Zanin, Ledezma, Galarsi, \& De Bortoli, 2010).

Las tareas de fluencia verbal, tanto FVS como FVF también han sido aplicadas a pobla- 
ciones infantiles. Los resultados de pruebas en niños se han utilizado en estudios sobre trastorno por déficit de atención, síndrome de Turner, dislexia del desarrollo, entre otros. La prueba de FVF es especialmente sensible para detectar niños con Trastorno por Déficit de Atención con Hiperactividad (Hurks et al., 2004).

Existen diversos estudios sobre poblaciones infantiles en tareas de fluencia, la mayoría de ellos realizados en niños españoles (Nieto, Galtier, Barroso, \& Espinosa, 2008), pero también es posible encontrar algunas investigaciones para la Argentina (Arán-Filippetti, 2011; García Coni \& Vivas, 2014; Marino \& Díaz-Fajreldines, 2011). El mayor interés sobre los estudios de fluencia verbal en niños se debe a que estas tareas evalúan mecanismos cognitivos que aún se encuentran en desarrollo en los niños, dando una perspectiva más clara sobre la ontogénesis de estos mecanismos. En este sentido, las variables sociodemográficas son extremadamente importantes ya que arrojan luz sobre los períodos de desarrollo de las habilidades cognitivas específicas. En las investigaciones con niños se ha encontrado que la edad es sin lugar a dudas la variable más relevante. La fluencia verbal aumenta en la infancia y en la adolescencia (Matute, Rosselli, Ardila, \& Morales, 2004). La FVS se incrementa principalmente en niños de 8 a 11 años de edad (Lozano Gutiérrez \& Ostrosky-Solís, 2006) y parece estar relacionada con un desarrollo progresivo en la semántica del lenguaje. Asimismo, el incremento en las tareas de FVF es menor que en las de FVS (Koren, Kofman, \& Berger, 2005).

A pesar de lo anteriormente expuesto, aún no es posible indicar en qué momento el desempeño de los niños en estos tests se equipara con el de los adultos. Algunas de las investigaciones reportan que a los 10 años los niños logran un rendimiento similar al de los adultos (Anderson, Northam, Hendy, \& Wrenall, 2001), en tanto que otros indican que recién en la adolescencia y adultez temprana (Klenberg, Korkman, \& Lahti-Nuuttila, 2001). Algunas investigaciones disocian el desempeño de los niños observado en FVS y FVF. El rendimiento de los niños en FVF se emparejaría con el de los adultos con posterio- ridad a lo observado con la FVS, que alcanzaría valores similares a los de la edad adulta entre los 14 y 15 años (Matute, et al., 2004). Otros estudios realizados en poblaciones infantiles han correlacionado positivamente el desempeño de los sujetos, aún en edad escolar, con el nivel educativo alcanzado por los padres (Ardila, Rosselli, Matute, \& Guajardo, 2005).

Dado que no existe aún información precisa acerca del patrón de desempeño de niños en edad escolar en tareas de fluencia verbal, el objetivo del presente trabajo es conocer el patrón de desempeño en FVF y FVS de forma evolutiva de niños hablantes del español de Argentina en edad escolar primaria. Para la FVS se obtuvieron datos de varias categorías semánticas (animales, frutas y verduras, partes del cuerpo, ropa, instrumentos musicales y medios de transporte) a fin de obtener una medición más precisa de la progresión semántica del lenguaje. En lo relativo a la FVF se obtuvo información de los fonemas $\mathrm{F}, \mathrm{A}, \mathrm{S}$, ya que estas fluencias constituyen una herramienta de pesquisa utilizada universalmente en la evaluación clínica.

\section{Metodología}

\section{Participantes}

Participaron de la investigación un total de 86 niños. La muestra está compuesta por 32 niños de $3^{\circ}$ grado ( $40.6 \%$ de hombres) con una media de edad de $8.58(D E=0.38), 25$ alumnos de $5^{\circ}$ grado ( $40 \%$ de hombres) con una media de edad de $10.70(D E=0.41)$; y 29 de $7^{\circ}$ grado $(44.8 \%$ de hombres) con una media de edad de 12.54 ( $D E$ $=0.43$ ). Todos los participantes evaluados eran hablantes nativos de español, no presentaban ningún déficit sensorial, alteraciones neurológicas ni trastornos del aprendizaje diagnosticados al momento de la evaluación. La totalidad de los sujetos evaluados concurría a una escuela privada de nivel socioeconómico medio de la Ciudad Autónoma de Buenos Aires. Para establecer el nivel socioeconómico de los participantes, se tuvo en cuenta el nivel educativo y las ocupaciones de los padres. Según Sautú (1991), al menos uno de los padres debe haber alcanzado el nivel 
educativo terciario o universitario para incluirse en este grupo socioeconómico. Este criterio fue considerado para la selección de la muestra. Los participantes respondieron las pruebas de manera voluntaria y se contó no solo con el aval de los directivos y los padres de los niños, sino también con el de la Dirección General de Planeamiento Educativo del Gobierno de la Ciudad de Buenos Aires para realizar la evaluación.

\section{Procedimiento}

Los alumnos de cada grado fueron evaluados con una tarea de FVF y una tarea de FVS en una única sesión de manera individual por uno de los investigadores durante el mes de septiembre de 2014. La evaluación se realizó en horario de clase en una sala provista por la institución para tal fin.

Ambas pruebas se tomaron de manera conjunta, los participantes primero respondían la tarea de FVF y luego la de FVS.

La consigna para la primera tarea era la siguiente: "Te pido que digas en un minuto todas las palabras que se te ocurran, no nombres propios, que empiecen con el siguiente fonema, por ejemplo M". Los participantes respondieron a los fonemas F, A, S. Antes de la evaluación, se constataba que los niños hubieran comprendido la consigna y se les proponían dos fonemas de prueba $\mathrm{P}$ y $\mathrm{M}$, una vez que nos asegurábamos de que podían realizar la tarea se procedía a la evaluación. Una vez finalizada la evaluación de FVF, se procedía a la evaluación de FVS, para tal fin se administraba la siguiente consigna: "Ahora, te voy a pedir que me digas en un minuto todas las palabras que se te ocurran de las distintas categorías que te voy a decir, por ejemplo profesiones". Los niños respondieron categorías del dominio de seres vivos (SV) (animales, frutas/verduras y partes del cuerpo) y del dominio de objetos inanimados (OI) (ropa, instrumentos musicales y medios de transporte). A su vez, se les propusieron dos categorías de prueba (profesiones y deportes) para tener certeza de que habían comprendido la consigna. Las respuestas de los participantes para ambas tareas fueron registradas con un grabador digital.
Una persona se ocupó de desgrabar las respuestas de manera idéntica a las producciones de los niños. No obstante, sólo se consideraron como respuestas válidas para las fluencias semánticas los nombres de los ejemplares de las categorías. Se eliminaron las palabras que indican un mismo ejemplar (por ejemplo, león - leona), no se aceptaron las categorías superordinadas (por ejemplo, peces). Las variaciones de una misma respuesta se agruparon en una única (por ejemplo, manzanas verdes, manzanas rojas se unificaron en manzanas). Se quitaron los errores dentro de una categoría específica (por ejemplo perro de la categoría instrumentos musicales). Para la fluencia fonológica no se contabilizaron los nombres propios ni las familias de palabras (perra, perro, perrito, etc.). Tanto en FVS como en FVF se eliminaron las palabras repetidas.

\section{Análisis Estadístico}

A fin de identificar posibles diferencias en el desempeño en tareas de fluencia semántica y fonológica de niños en edad escolar hablantes del español de Argentina se realizó un análisis mixto de varianza según el modelo ANOVA $2 \times 3$, tomando como variables intrasujetos la fluencia (fonológica y semántica) y como variable intersujeto el grado $\left(3^{\circ}, 5^{\circ}\right.$ y $\left.7^{\circ}\right)$.

Un segundo análisis se realizó a fin de identificar posibles diferencias en el procesamiento de los dominios semánticos de SV y OI. Para esto se aplicó otro análisis mixto de varianza ANOVA $2 \times 3$. Se tomó el dominio (SV vs. OI) como variable intrasujeto y el grado $\left(3^{\circ}, 5^{\circ}\right.$ y $\left.7^{\circ}\right)$ como variable intersujeto. Se calculó para ambos análisis el tamaño del efecto a partir del cálculo de Eta cuadrado parcial $\left(\eta^{2}\right)$.

\section{Resultados}

A continuación, en la Tabla 1 se muestra el desempeño en las tareas de fluencia fonológica y semántica en los diferentes grados. Los puntajes presentados en la misma son: la media del promedio de ejemplares evocados por los participantes para cada categoría semántica evaluada en la tarea de fluidez semántica y para cada fonema evaluado en la tarea de fluidez fonológica. 
Tabla 1

Media y Desvío Estándar para el Promedio de la Fluencia Fonológica y Semántica en Cada Uno de los Grados

\begin{tabular}{lcccc}
\hline & \multicolumn{3}{c}{ Fluencia } \\
\cline { 2 - 5 } Grado & \multicolumn{2}{c}{ Semántica } & \multicolumn{2}{c}{ Fonológica } \\
\cline { 2 - 5 } & $M$ & $D E$ & $M$ & $D E$ \\
\hline 3er grado & 11.17 & 1.99 & 4.77 & 1.72 \\
5to grado & 13.06 & 2.46 & 7.32 & 2.42 \\
7mo grado & 13.69 & 2.29 & 7.92 & 2.05 \\
\hline
\end{tabular}

Se calculó para cada participante el promedio de la cantidad de ejemplares evocados para las categorías de fluencia semántica (correspondientes a la fluencia de animales, frutas y verduras, partes del cuerpo, transportes, instrumentos musicales y ropa) y el promedio de ejemplares evocados para cada fonema de fluencia fonológica (correspondientes a la fluencia de /f/, /a/ y /s/). El primer análisis da cuenta de diferencias estadísticamente significativas en el desempeño en las tareas de fluencia a favor de la recupera- ción semántica en general, $F_{(1,83)}=502.30$; MSE $=3.02 ; p<.001 ; \eta 2=.86$. También indica diferencias significativas para grado, $F_{(2,83)}=22.00$; $\mathrm{MSE}=6.19 ; p<.001 ; \eta 2=.35$. El análisis posthoc, empleando el método de Bonferroni, indica que los alumnos de $7^{\circ}$ no difieren significativamente de los alumnos de $5^{\circ}$, pero ambos grados difieren significativamente $(p<.001)$ de los de $3^{\circ}$. Asimismo, el análisis no detectó efectos de interacción, $F_{(2,83)}=0.68 ; \mathrm{MSE}=3.02 ; p=.51$; $\eta 2=.02$.

Tabla 2

Medias y Desvíos Estándar en el Dominio de SV y de OI en Cada Uno de los Grados Evaluados

\begin{tabular}{lcccc}
\hline & \multicolumn{5}{c}{ Dominio semántico } \\
\cline { 2 - 5 } Grado & \multicolumn{2}{c}{$\mathrm{SV}$} & & OI \\
\cline { 2 - 5 } & $M$ & $D E$ & $M$ & DE \\
\hline 3er grado & 13.17 & 2.37 & 9.17 & 2.02 \\
5to grado & 15.76 & 3.32 & 10.36 & 2.30 \\
7 mo grado & 16.80 & 3.32 & 10.59 & 1.97
\end{tabular}

Los resultados del segundo análisis se presentan en la Tabla 2 y muestran el desempeño en las tareas de fluencia semántica para los diferentes dominios en los diferentes grados. Se calculó para cada participante el promedio de la cantidad de ejemplares evocados para cada dominio. El dominio SV está conformado por la categorías de animales, frutas y verduras y partes del cuerpo, mientras que el dominio de OI está conformado por las categorías transportes, ropa e ins- trumentos musicales. Este análisis muestra un efecto significativo para el dominio semántico, $F_{(1.83)}=341.228 ; \operatorname{MSE}=3.381 ; p<.001 ; \eta 2=$ .804. Los niños activan mayor cantidad de ejemplares del dominio de SV que del de OI. También hay diferencias estadísticamente significativas para grado, $F_{(2.83)}=10.584$; $\mathrm{MSE}=9.992$; $p<.001 ; \eta 2=.203$. La cantidad de ejemplares recuperados aumenta en los alumnos de los grados superiores. El análisis post hoc (empleando 
el método de Bonferroni) permite ver que los niños de $3^{\circ}$ grado se disocian significativamente de los de $5^{\circ}(p<.01)$ y de $7^{\circ}(p<.001)$. En tanto que los alumnos de $5^{\circ}$ y de $7^{\circ}$ no se diferencian en su desempeño.

Por otro lado, la interacción dominio y grado también es significativa, $F_{(2.83)}=5.693$; MSE $=3.381 ; p<.01 ; \eta 2=.121$. Al igual que en el análisis según grado, los alumnos de $3^{\circ}$ grado sólo se disocian de los de $5^{\circ}(p<.05)$ y $7^{\circ}(p$ $<.05)$ en la cantidad de ejemplares recuperados para el dominio de SV. En tanto que los alumnos de $5^{\circ}$ y de $7^{\circ}$ no se diferencian en la cantidad de ejemplares recuperados para este dominio.

\section{Discusión}

El objetivo de este trabajo fue obtener información acerca del desempeño en tareas de fluidez verbal de niños argentinos en edad escolar. Con esta finalidad se obtuvieron datos para FVS (animales, frutas/verduras, partes del cuerpo, medios de transporte, instrumentos musicales y ropa) y para FVF (prueba FAS).

Al igual que en los estudios previos en el tema, los datos obtenidos en la población de Argentina muestran que los niños en edad escolar mejoran su desempeño con el incremento de la edad y alcanzan un mejor rendimiento en tareas de FVS que FVF (Brocki \& Bohlin, 2004; García et al., 2012; García Coni \& Vivas, 2014; Matute et al., 2004; Valencia et al., 2000). En la muestra evaluada, se observa un incremento en la cantidad de respuestas correctas, para ambas tareas, de $3^{\circ}$ a $5^{\circ}$ grado. A partir de $5^{\circ}$ grado la mejora en el rendimiento en ambas tareas deja de ser estadísticamente significativa. En lo que a la FVS respecta, la media de respuestas obtenida para la categoría de animales en los niños de $5^{\circ}$ grado es equivalente a la reportada para una muestra de adultos argentinos menores de 45 años con estudios primarios incompletos (Butman, Allegri, Harris, \& Drake, 2000). Por el contrario, en la FVF si bien no hay diferencias significativas entre $5^{\circ}$ y $7^{\circ}$, se observa un incremento en la cantidad de respuestas dadas para los fonemas $/ \mathrm{a} / \mathrm{y} / \mathrm{s} /$. A diferencia de lo que ocurre en los niños en la FVS, los datos no son equiparables con los reportados para nuestra población en adultos (Butman et al., 2000). Esto podría deberse a que en la población de adultos se evaluó el fonema /p/. No obstante, y teniendo en cuenta que el desempeño de los niños evaluados en nuestra muestra es menor, consideramos que la diferencia encontrada en el desempeño en ambas fluencias verbales puede ser explicada por el nivel de maduración de los sistemas cognitivos involucrados en cada una de las tareas. En la FVS se debe recuperar ejemplares de una única categoría previamente dada por el evaluador (por ejemplo, animales). Esto provoca que la búsqueda se limite a la recuperación de ítems pertenecientes a una categoría semántica familiar para los niños. Por lo tanto, en la tarea de FVS, los mecanismos de función ejecutiva, que aún se encuentran en desarrollo en niños de esta edad, no tienen la misma incidencia que en las tareas de FVF en las que la búsqueda que debe realizar el evaluado no se limita a una única categoría y debe recorrer una mayor cantidad de subcategorías. Para llevar a cabo esta tarea, los niños se enfrentan a la necesidad de realizar una búsqueda intercategorial. Además, deben aplicar un criterio de búsqueda que no es únicamente semántico, como ocurre en la FVS, sino también de organización lingüística de los ítems. Por lo anteriormente dicho, la tarea de FVF resulta más difícil para los niños dado que deben activar mecanismos de inhibición para la ejecución de la tarea y así evitar la producción de formas relacionadas semánticamente incorrectas para la tarea (Arán-Filippetti, 2011). Estos mecanismos de búsqueda estratégicos e inhibición, sustentados por el lóbulo frontal, parecerían automatizarse tanto durante el desarrollo cognitivo como también con el proceso de escolarización formal de los niños. Como consecuencia, los niños mayores realizan mejores búsquedas que los más pequeños que se reflejan tanto en el incremento de la cantidad de ítems producidos como también en la precisión de los ítems seleccionados. Es decir, los evaluados de $7^{\circ}$ presentan, en tareas de FVF, menor número de intrusiones, por ejemplo, de nombres propios.

En el marco de evaluaciones neuropsicológicas infantiles, las tareas de fluidez fonológica 
brindan medidas confiables en la detección de trastornos atencionales, puesto que esta tarea exige que el evaluado mantenga la atención sostenida por períodos prolongados de tiempo mientras implementa diferentes estrategias de búsqueda de vocabulario. Los niños que padecen Trastorno por déficit de atención e hiperactividad (TDAH) suelen tener resultados muy por debajo de la media en estas tareas, ya que el período durante el cual sostienen la atención es muy reducido (Grodzinsky \& Diamond, 1992). Asimismo, esta tarea también resulta importante en la evaluación de niños con dislexia ya que se trata de una medida útil para el diagnóstico y el seguimiento del tratamiento implementado (Cohen, Morgan, Vaughn, Riccio, \& Hall, 1999; Reiter, Tucha, \& Lange, 2005).

A diferencia de los trabajos previos realizados para nuestra población, que evalúan el desempeño en los niños en una única categoría semántica (específicamente la de animales), en nuestro trabajo testeamos varias categorías pertenecientes al dominio de SV y otras del de OI a fin de poder identificar diferencias semánticas en el procesamiento infantil.

Nuestros datos muestran diferencias de procesamiento a favor del dominio de SV. En consonancia con los resultados obtenidos por Valencia et al. (2000), las categorías de este dominio son categorías naturales y, por ende, para su establecimiento podrían necesitarse menos años de escolarización. Los datos muestran que los niños de $3^{\circ}$ grado difieren significativamente de los de $5^{\circ}$ y $7^{\circ}$ sólo en el dominio de $\mathrm{SV}$, pero esto no sucede entre los niños de $5^{\circ}$ y $7^{\circ}$ que muestran un rendimiento equivalente. Sin embargo, para el dominio de OI no se detectan diferencias entre los tres grupos que muestran un rendimiento más bajo que en el caso del otro dominio semántico.

Retomando lo previamente planteado en la introducción de este trabajo, una de las cuestiones que aún permanece en debate acerca de en qué momento del desarrollo evolutivo los niños alcanzan un rendimiento similar al de los adultos en tareas de fluencia verbal, nuestros datos nos permiten observar que los niños de $5^{\circ}$ grado alcanzan un rendimiento equiparable al de adultos jóvenes con primario incompleto en la tarea de fluidez semántica para la categoría animales (única categoría contemplada en las investigaciones de adultos). Dado que nuestra muestra está compuesta por tres grupos no consecutivos, los resultados no nos permiten saber con certeza en qué momento del desarrollo los niños equiparan su capacidad de recuperar ejemplares desde su almacén semántico con la de los adultos. Por lo tanto, será necesario ampliar la muestra e incluir datos de rendimiento de niños que asisten a $4^{\circ}$ grado para establecer con mayor precisión el momento de culminación del desarrollo semántico para la categoría de animales.

En relación con la tarea de fluidez fonológica, cabe señalar que los niños de nuestra muestra no alcanzan a equipararse con el rendimiento de sujetos adultos evaluados en otros trabajos realizados en nuestra población. Por ende, hasta los 12 años de edad las habilidades cognitivas involucradas en las tareas de fluidez fonológica aún seguirían desarrollándose. Para poder tener un conocimiento más certero del momento en el cuál niños y adultos se emparejan en cuanto a rendimiento en tareas de fluidez fonológica, es necesario ampliar la muestra y evaluar niños de mayor edad y adolescentes.

La disociación encontrada en el desempeño entre FVS y FVF está en concordancia con los hallazgos de Hurks et al. (2006) y Matute et al. (2004) que señalan que las habilidades de fluidez verbal semántica y fonológica no se desarrollan conjuntamente.

Por otra parte, dado que no contamos con una base de datos normatizada para la población de niños de nuestro país, el presente trabajo es de suma relevancia. Esta investigación forma parte de un proyecto de normatización de fluidez semántica de diversas categorías semánticas en niños (participantes de edad preescolar y primaria), adolescentes (estudiantes de secundaria) y adultos.

Este trabajo, no obstante, tiene ciertas limitaciones. Por un lado, la población estudiada, puesto que sólo se limita a una población media que asiste a una escuela privada. En función de esto se deberá ampliar la muestra evaluando alumnos que asistan a escuelas públicas. Asimismo, el nivel de escolaridad de los padres 
de nuestra muestra indica que pertenecen a una clase media por lo que también se deberá tener información de otras clases socioeconómicas de nuestro país (baja y alta).

Otra limitación, teniendo en cuenta la diversidad de la población del país, es que nuestra muestra sólo representa a la región del Ciudad Autónoma de Buenos Aires y, posiblemente, a parte de la población de la Provincia de Buenos Aires, por lo tanto se requiere evaluar a alumnos que se encuentren en el resto de las regiones de nuestro país (norte y sur) a fin de identificar posibles disociaciones en función de la región.

En este trabajo el análisis se centró en identificar posibles diferencias en función de la cantidad de ejemplares recuperados. Como posible línea de investigación futura se prodría realizar un análisis de clusters (Troyer et al., 1998) a fin de conocer la organización semántica de cada una de las categorías evaluadas con las fluencias.

\section{Referencias}

Albanese, E., Capitani, E., Barbarotto, R., \& Laiacona, M. (2000). Semantic category dissociations, familiarity and gender. Cortex, 36, 733-746.

Anderson, V., Northam, E., Hendy, J., \& Wrenall, J. (2001). Developmental Neuropsychology: A clinical approach. New York: Psychology Press.

Arán-Filippetti, V. (2011). Fluidez verbal según tipo de tarea, intervalo de tiempo y estrato socioeconómico, en niños escolarizados. Anales de Psicología, 27(3), 816-826.

Ardila, A., Rosselli, M., Matute, E., \& Guajardo, G. (2005). The influence of parents educational level on the development of executive functions. Developmental Neuropsychology, 28, 539-560. doi:10.1207/s15326942dn2801_5

Binetti, G., Magni, E., Cappa, S. F., Padovani, A., Bianchetti, A., \& Trabucchi, M. (1995). Semantic memory in Alzheimer's disease: An analysis of category fluency. Journal of Clinical and Experimental Neuropsychology, 17, 82-89.

Brocki, K. C., \& Bohlin, G. (2004). Executive functions in children aged 6 to 13: A dimensional and developmental study. Developmental Neuropsychology, 26, 571-593. doi:10.1207/ s15326942dn2602_3
Buriel, Y., Gramunt, N., Bohm, P., Rodes, E., \& Pena-Casanova, J. (2004). Verbal fluency: Preliminary normative data in a Spanish sample of young adults (20-49 years of age). Neurología, 19(4), 153-159.

Butman, J., Allegri, R. F., Harris, P., \& Drake, M. (2000). Fluencia verbal en español. Medicina, 60, 561-564.

Capitani, E., Laiacona, M., \& Barbarotto, R. (1999). Gender affects Word retrieval of certain categories in semantic fluency tasks. Cortex, 35, 273278. doi:10.1016/S0010-9452(08)70800-1

Casals-Coll, M., Sánchez-Benavides, G., Quintana, M., Manero, R. M., Rognoni, T., Calvo, L., ...Peña-Casanova, J. (2013). Estudios normativos españoles en población adulta joven (proyecto NEURONORMA jóvenes): normas para los test de fluencia verbal. Neurología, 28(1), 33-40.

Cohen, M. J., Morgan, A. M., Vaughn, M., Riccio, C. A., \& Hall, J. (1999). Verbal fluency in children: Developmental issues and differential validity in distinguishing children with attention-deficit hyperactivity disorder and two subtypes of dyslexia. Archives of Clinical Neuropsychology, 14(5), 433-443. doi:10.1016/ S0887-6177(98)00038-9

Comesaña, A., \& García Coni, A. (2013). Análisis de Tareas de Fluidez Verbal en Enfermos de Alzheimer y Adultos Sanos. Temas em Psicologia, 21(1), 269-281. doi:10.9788/ TP2013.1-19

Chertkow, H., \& Bub, D. (1990). Semantic memory loss in dementia of Alzheimer's type. What do various measures measure? Brain, 113, 397-417.

Fernández, A. L., Marino, J. C., \& Alderete, A. M. (2004). Valores normativos en la prueba de fluidez verbal-animales sobre una muestra de 251 adultos argentinos. Revista Argentina de Neuropsicología, 4, 12-22.

Galaverna, F. S., Marino, J. C., \& Abraham, M. (2008). Estudio Comparativo de la Organización Semántica entre Sujetos con Trastorno Esquizofrénico y Sujetos Normales. Revista Neuropsicología, Neuropsiquiatría y Neurociencias, 8(2), 59-71.

García, E., Rodríguez, C., Martín, R., Jiménez, J. E., Hernández, S., \& Díaz, A. (2012). Test de Fluidez Verbal: datos normativos y desarrollo evolu- 
tivo en el alumnado de primaria. European Journal of Education and Psychology, 5(1), 53-64.

García Coni, A., \& Vivas, J. (2014). Estrategias ejecutivas de búsqueda, recuperación y cambio en la fluidez verbal. Evaluar, 14, 15-42.

Grodzinsky, G. M., \& Diamond, R. (1992). Frontal lobe functioning in boys with attention-deficit hyperactivity disorder. Developmental Neuropsychology, 8, 427-445. doi:10.1080/87565649209540536

Halpern, D. F. (1992). Sex differences in cognitive abilities ( $2^{\text {nd }}$ ed.). Hillsdale, $\mathrm{NJ}$ : Erlbaum.

Henry, J. D., \& Crawford, J. R. (2004). A metaanalytic of verbal fluency performance following focal cortical lesions. Neuropsychology, 18, 284-295. doi:10.1037/0894-4105.18.2.284

Henry, J. D., Crawford, J. R., \& Phillips, L. H. (2004). Verbal fluency performance in dementia of the Alzheimer's type: A meta-analysis. Neuropsychologia, 42, 1212-1222.

Hirshorn,E., \& Thompson-Schill, S.(2006). Role of the left inferior frontal gyrus in covert word retrieval: Neural correlates of switching during verbal fluency. Neuropsychologia, 44, 2547-2557. doi:10.1016/j.neuropsychologia.2006.03.035

Hodges, J. R., \& Patterson, K. (1997). Semantic memory disorders. Trends in Cognitive Sciences, 1(2), 68-72. doi:10.1016/S13646613(97)01022-X

Hurks, P. P. M., Hendriksen, J. G. M., Vles, J. S. H., Kalff, A. C., Feron, F. J. M., Kroes, M., ...Jolles, J. (2004). Verbal fluency over time as a measure of automatic and controlled processing in children with ADHD. Brain and Cognition, 55, 535 544. doi:10.1016/j.bandc.2004.03.003

Hurks, P. P. M., Vles, J. S. H., Hendriksen, J. G. M., Kalff, A. C., Feron, F. J. M., Kroes, M., ...Jolles, J. (2006). Semantic category fluency versus initial letter fluency over 60 seconds as a measure of automatic and controlled processing in healthy school-aged children. Journal of Clinical and Experimental Neuropsychology, 28, 684-695. doi:http://dx.doi. org/10.1080/13803390590954191

Kempler, D., Teng, E. L., Dick, M., Taussig, I. M., \& Davis, D. S. (1998). The effects of age, education, and ethnicity on verbal fluency. Journal of the International Neuropsychological Society, 4, 531-538. doi:http://dx.doi.org/10.1017/ S1355617798466013
Klenberg, L., Korkman, M., \& Lahti-Nuuttila, P. (2001). Differential development of attention and executive functions in 3 to 12 years-old Finnish children. Developmental Neuropsychology, 20, 407-428. doi:http://dx.doi.org/10.1207/ S15326942DN2001_6

Koren, R., Kofman, O., \& Berger, A. (2005). Analysis of word clustering in verbal fluency of school-aged children. Archives of Clinical Neuropsychology, 20, 1087-1104. doi:http://dx.doi. org/10.1016/j.acn.2005.06.012

Laws, K. R. (1999). Gender afects latencies for naming living and nonliving things: implications for familiarity. Cortex, 35, 729-733.

Laws, K. R. (2004). Sex differences in lexical size across semantic categories. Personality and Invidual Differences, 36, 23-32. doi:http://dx.doi. org/10.1016/S0191-8869(03)00048-5

Lezak, M. D., Howieson, D. B., \& Loring, D. W. (2004). Neuropsychological assessment. New York: Oxford University Press.

Lozano Gutiérrez, A., \& Ostrosky-Solís, F. (2006). Efecto de la edad y la escolaridad en la fluidez verbal semántica: datos normativos en población hispanohablante. Revista Mexicana de Psicología, 23(1), 37-44.

Marino, J., \& Alderete, A. M. (2010). Valores normativos de pruebas de fluidez verbal categoriales, fonológicas, gramaticales y combinadas y análisis comparativo de la capacidad de iniciación. Revista Neuropsicología, Neuropsiquiatría y Neurociencias, 10(1), 79-93.

Marino, J., \& Díaz-Fajreldines, H. (2011). Pruebas de fluidez verbal categoriales, fonológicas y gramaticales en la infancia: factores ejecutivos y semánticos. Revista Chilena de Neuropsicología, 6(1), 49-56.

Marra, C., Ferraccioli, M., \& Gainotti, G. (2007). Gender-related dissociations of categorical fluency in normal subjects and in subjects with Alzheimer's disease. Neuropsychology, 21(2), 207-211. doi:http://dx.doi.org/10.1037/08944105.21.2.207

Matute, E., Rosselli, M., Ardila, A., \& Morales, L. (2004). Verbal and non-verbal fluency in Spanish speaking children. Developmental Neuropsychology, 26, 647-660.

McKenna, P., \& Parry, R. (1994). Category-specificity in the naming of natural and man-made 
objects. Neuropsychological Rehabilitation, 4, 255-281. doi:10.1080/09602019408401461

Nieto, A., Galtier, I., Barroso, J., \& Espinosa, G. (2008). Fluencia verbal en niños españoles en edad escolar: estudio normativo piloto y análisis de las estrategias organizativas. Revista Neurologica, 46(1), 2-6.

Ollari, A., \& Diez, S. (2005). Neurobiología del envejecimiento. In O. Mangone, R. Allegri, R. Arizaga, \& J. Ollari (Eds.), Demencia: enfoque multidisciplinario (pp. 1-22). Buenos Aires: Polemos.

Ostrosky-Solís, F., \& Ardila, A. (1991). Un esquema de diagnóstico neuropsicológico: efectos socioculturales y su aplicación en el diagnóstico del daño cerebral. Revista Mexicana de Salud Mental, 14(4), 17-23.

Pekkala, S., Goral, M., Hyun, J., Obler, L. K., Erkinjuntti, T., \& \& Albert, M. (2009). Semantic verbal fluency in two contrasting languages. Clin Linguist Phon., 23(6), 431-445. doi:http:// dx.doi.org/10.1080/02699200902839800

Ramírez, M., Ostrosky-Solís, F., Fernández, A., \& Ardila-Ardila, A. (2005). Fluidez verbal semántica en hispanohablantes: un análisis comparativo. Revista de Neurología, 41, 463-468.

Ratcliff, G., Ganguli, M., Chandra, V., Sharma, S., Belle, S., Seaberg, E., \& Pandav, R. (1998). Effects of literacy and education on measures of word fluency. Brain and Language, 61, 115-122. doi:http://dx.doi.org/10.1006/brln.1997.1858

Reiter, A., Tucha, O., \& Lange, K. W. (2005). Executive functions in children with dyslexia. Dyslexia, 11(2), 116-131. doi:http://dx.doi. org/10.1002/dys.289

Rodríguez-Aranda, C., \& Martinussen, M. (2006). Age-related differences in performance of phonemic verbal fluency measured by Controlled Oral Word Association Task (COWAT): A meta-analytic study. Developmental Neuropsychology, 30, 697-717.

Rosser, A., \& Hodges, J. R. (1994). Initial letter and semantic category fluency in Alzheimer's disease, Huntington's disease, and progressive supranuclear palsy. Journal of Neurology, Neurosurgery and Psychiatry, 57, 1389-1394.

Sautú, R. (1991). Oportunidades ocupacionales diferenciales por sexo en Argentina: 1970/1980. Paper presented at the Estudios del Trabajo, Buenos Aires, Argentina.
Soriano, F., Fumagalli, J., Shalóm, D., Carden, J., Borovinsky, G., Manes, F., \& Martínez-Cuitiño, M. (2015). Sex differences in a semantic fluency task. East European Journal of Psycholinguistic 2(1), 134-140.

Stuss, D. T., Alexander, M. P., Hamer, L., Palumbo, C., Dempster, R., Binns, M., ...Izukawa, D. (1998). The effects of focal anterior and posterior brain lesions on verbal fluency. Journal of the International Neuropsychological Society, 4, 265-278.

Tombaugh, T. N., Kozak, J., \& Rees, L. (1999). Normative data stratified by age and education for two measures of verbal fluency: FAS and animal naming. Archives of Clinical Neuropsychology, 14(2), 167-177. doi:http://dx.doi.org/10.1093/ $\operatorname{arclin} / 14.2 .167$

Troyer, A., Moscovitch, M., Winocur, G., Alexander, M. P., \& Stuss, D. T. (1998). Clustering and switching on verbal fluency: The effects of focal frontal and temporal lobes lesions. Neuropsychologia, 36(6), 499-504. doi:http://dx.doi. org/10.1016/S0028-3932(97)00152-8

Valencia, N. J., Laserna, J. A., Pérez-García, M., Orozco, C., Miñán, M., Garrido, C., ...Morente, G. (2000). Influencia de la escolaridad y el sexo sobre la ejecución en el fas, nombrar animales y nombrar frutas. Psicología Conductual, 8(2), 283-295.

Villodre, R., Sánchez-Alfonso, A., Brines, L., Núñez, A. B., Chirivella, J., Ferri, J., \& Noé, E. (2006). Verbal fluency tasks in a Spanish sample of young adults (20-49 years of age): normative data of clustering and switching strategies. $\mathrm{Neu}$ rología, 21(3), 124-130.

Zanin, L., Ledezma, C., Galarsi, F., \& De Bortoli, M. A. (2010). Fluidez verbal en una muestra de 227 sujetos de la región Cuyo (Argentina). Fundamentos en Humanidades, 21(1), 207-219. 